\title{
The Correlation Between Computed Tomography Volumetry and Prognosis of Advanced Gastric Cancer Treated with Neoadjuvant Chemotherapy
}

This article was published in the following Dove Press journal: Cancer Management and Research

\author{
Chao Chen' \\ Hao Dong' \\ Chunhui Shou' \\ Xiaoxiao Shi ${ }^{1}$ \\ Qing Zhang' \\ Xiaosun Liu' \\ Kankai Zhu' \\ Baishu Zhong ${ }^{2}$ \\ Jiren $\mathrm{Yu}^{\prime}$
}

'Department of Gastrointestinal Surgery, The First Affiliated Hospital, Zhejiang University School of Medicine, Hangzhou, People's Republic of China; ${ }^{2}$ Department of Radiology, The First Affiliated Hospital, Zhejiang University School of Medicine, Hangzhou, People's Republic of China
Correspondence: Jiren Yu

Department of Gastrointestinal Surgery, The First Affiliated Hospital, Zhejiang University School of Medicine, 79 Qingchun Road, Hangzhou 310003,

Zhejiang Province, People's Republic of China

Tel $+8657|87236| 47$

Fax +8657187072577

Email yujr0909@zju.edu.cn
Purpose: To investigate the feasibility and utility of computer tomography (CT) volumetry in evaluating the tumor response to neoadjuvant chemotherapy (NAC) in advanced gastric cancer (AGC) patients.

Patients and Methods: One hundred and seventeen Patients with AGC who received NAC followed by R0 resection between January 2006 and December 2012 were included. Tumor volumes were quantified using OsiriX software. The volume reduction rate (VRR) was calculated as follows: VRR $=[$ (pre-chemotherapy total volume) - (post-chemotherapy total volume $)] /($ pre-chemotherapy total volume $) \times 100 \%$. The optimal cut-off VRR for differentiating favorable from unfavorable prognosis was determined by receiver operating characteristic (ROC) analysis. Overall survival was calculated using Kaplan-Meier analysis and values were compared using the Log-rank test. Multivariate analysis was determined by the Cox proportional regression model.

Results: The optimal cut-off VRR was $31.95 \%$ according to ROC analysis, with a sensitivity of $70.4 \%$ and a specificity of $71.7 \%$. Based on the cut-off VRR, patients were divided into the VRR-High (VRR $\geq 31.95 \%, \mathrm{n}=63$ ) and VRR-Low (VRR < 31.95\%, $\mathrm{n}=54$ ) groups. The VRR-Low group exhibited a worse prognosis than that of the VRR-High group (HR, 2.85; 95\% CI, 1.69-4.82, P < 0.001), with 3-year survival rates of $40.7 \%$ and $79.4 \%$, and 5 -year survival rates of $31.5 \%$ and $63.5 \%$, respectively.

Conclusion: CT volumetry is a feasible and reliable method for assessing the tumor response to NAC in patients with AGC.

Keywords: advanced gastric cancer, neoadjuvant chemotherapy, computed tomography volumetry

\section{Introduction}

Gastric cancer is the third-leading cause of cancer-related mortality worldwide, accounting for about 754,000 deaths annually. ${ }^{1}$ Although the 5-year overall survival (OS) rate of advanced gastric cancer (AGC) has improved from $23 \%$ to $40 \%$, the prognosis of AGC remains unsatisfactory. ${ }^{2-4}$ Neoadjuvant chemotherapy (NAC) is widely regarded as an effective multidisciplinary approach to AGC therapy, with evidence of improved survival of patients with AGC compared with surgery alone. $^{4-6}$ Furthermore, the adverse events associated with NAC in AGC were found to be tolerable and manageable, with a low perioperative morbidity. ${ }^{5,7}$ However, the methods used in these studies to evaluate the tumor response to NAC were not well established and remain controversial. 
Computed tomography (CT) is a common procedure for assessing the clinical stage of gastric cancer due to its convenient and non-invasive nature. The Response Evaluation Criteria in Solid Tumors (RECIST) are one of the most widely used methods for evaluating the response to chemotherapy in various tumors. ${ }^{8,9}$ According to the RECIST for gastric cancer, only lymph nodes with a shortaxis diameter greater than $15 \mathrm{~mm}$ or other metastatic lesions (such as hepatic lesions) are considered target lesions; ${ }^{9}$ primary gastric cancer lesions are deemed unsuitable targets because of their irregular shape, although the sensitivity and specificity of CT for detecting lymph node metastases vary in gastric cancer. One study reported a specificity of $99.8 \%$ and a positive predictive value of $98.6 \%$ for lymph nodes with a short-axis diameter greater than $15 \mathrm{~mm}$; however, the sensitivity of this test was only $22.5 \% .{ }^{10}$ Accordingly, the utility of the RECIST for evaluating the tumor response to NAC in patients with AGC might be limited. The Japanese Classification of Gastric Carcinoma-Response Assessment of Chemotherapy for Gastric Cancer (JCGC criteria) is based on morphological changes in the primary lesions, as determined using barium X-ray or endoscopic examinations, ${ }^{11}$ but it does not evaluate metastatic lesions such as lymph nodes. Furthermore, patients find the procedure inconvenient and are generally unwilling to undergo repeated endoscopic examinations.

Recent studies have suggested CT volumetry as a useful technique for tumor assessment in gastric cancer, ${ }^{12,13}$ yielding significantly greater accuracy in predicting $\mathrm{T}$ and $\mathrm{N}_{3}$ stages compared with conventional $\mathrm{CT},{ }^{14}$ with post-chemotherapy tumor volumes significantly correlated with tumor stage in patients with gastric cancer. ${ }^{13}$ Moreover, two small studies showed a significant association between $\mathrm{CT}$ volume reduction in primary gastric lesions after NAC and clinical outcomes. ${ }^{15,16}$ Here, we sought to investigate the feasibility and utility of $\mathrm{CT}$ volumetry for evaluating the tumor response to NAC.

\section{Patients and Methods}

\section{Ethics}

Ethics approval for this study was obtained from the Research Ethics Committee of the First Affiliated Hospital, College of Medicine, Zhejiang University. Patient consent was not required as this is a retrospective study. Patient data confidentiality was guaranteed. All procedures followed were in accordance with the ethical standards of the responsible committee on human experimentation (institutional and national) and with the Helsinki Declaration of 1964 and later versions.

\section{Patient Selection}

Patients with AGC who received NAC followed by R0 resection between January 2006 and December 2012 were considered for this study (Figure 1). The inclusion criteria were as follows: (1) pathologically confirmed gastric adenocarcinoma, (2) a primary tumor invading the serosa (T4a) or adjacent structures (T4b) with or without metastatic lymph nodes according to CT, (3) ambulatory males or females aged 18-80 years, (4) Eastern Cooperative Oncology Group score of 0-2, (5) life expectancy greater than 3 months, (6) and normal cardiac, hepatic, renal, and bone marrow function. The exclusion criteria included (1) distant metastasis (such as lymph nodes 13 and 16, liver, lung, brain, bone, and peritoneal metastases), (2) previous major stomach surgery, (3) previous cytotoxic chemotherapy, radiotherapy, target therapy, or immunotherapy for any tumor, (4) history of another malignancy except cured basal cell carcinoma of the skin and cured carcinoma in-situ of the uterine cervix, (5) and women who were pregnant, breastfeeding, or contemplating pregnancy.

\section{NAC Regimen and Surgery}

Chemotherapy regimens included XELOX $\left(130 \mathrm{mg} / \mathrm{m}^{2}\right.$ oxaliplatin as a 2-hrs infusion on day 1 , followed by $1000 \mathrm{mg} / \mathrm{m}^{2}$ capecitabine twice daily for 14 consecutive days), FOLFOX $\left(130 \mathrm{mg} / \mathrm{m}^{2}\right.$ oxaliplatin as a $2-\mathrm{hrs}$ infusion, $400 \mathrm{mg} / \mathrm{m}^{2}$ leucovorin, and a bolus of $400 \mathrm{mg} / \mathrm{m}^{2}$-fluorouracil on day 1 , followed by a 46-hrs infusion of $2400 \mathrm{mg} / \mathrm{m}^{2}$ 5-fluorouracil), and $\operatorname{SOX}\left(130 \mathrm{mg} / \mathrm{m}^{2}\right.$ oxaliplatin as a 2-hrs infusion on day 1 , followed by S-1 given orally twice daily for 2 weeks). The dose of S-1 was $80 \mathrm{mg} /$ day for a body surface area (BSA) $<1.25 \mathrm{~m}^{2}, 100 \mathrm{mg} /$ day for a BSA $\geq 1.25$ to $<1.5 \mathrm{~m}^{2}$, and $120 \mathrm{mg} /$ day for a BSA $\geq 1.5 \mathrm{~m}^{2}$. Chemotherapy was repeated every 3 weeks.

Surgery was performed after at least two cycles of chemotherapy. Distal, total gastrectomy, or combined resection was performed within 2 weeks after completion of the last cycle of NAC, depending on the location and extent of the primary tumor. D2 lymphadenectomy was conducted according to the criteria established by the Japanese Gastric Cancer Association. ${ }^{17}$ Postoperative chemotherapy was initiated at 4-6 weeks after surgery. The yield pathological (yp) TNM stage was assessed according to the criteria of the AJCC TNM staging system, 7 th edition. ${ }^{18}$ 


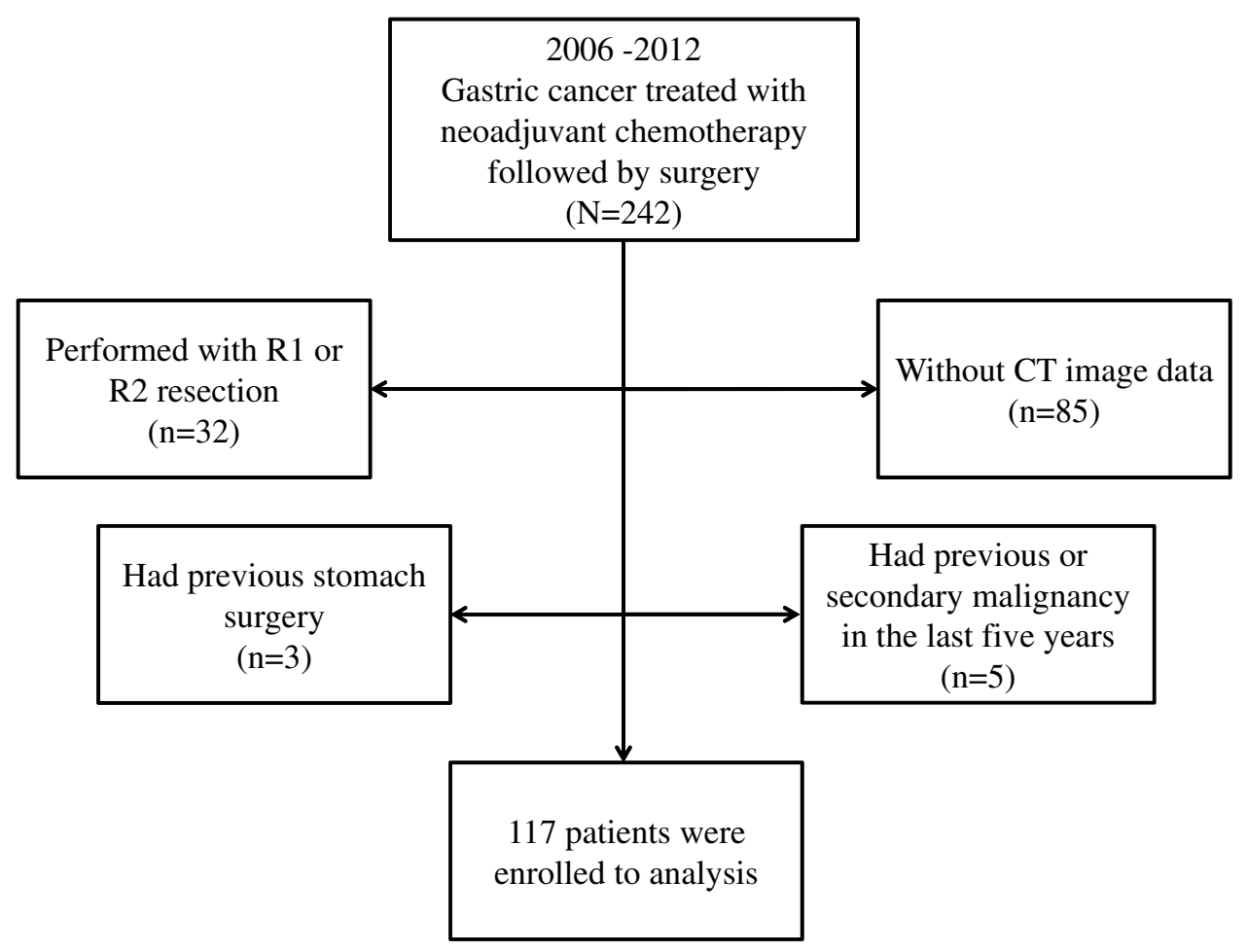

Figure I Flowchart shows the study enrollment. Of the 242 initial patients, II 7 were finally included.

\section{CT Acquisition and Analysis}

A baseline CT workup was obtained up to 2 weeks prior to the initiation of NAC. For response evaluation, CT was also performed after completing at least two cycles of NAC.

Patient preparation included oral administration of $1000 \mathrm{~mL}$ water before CT. Patients were placed in the supine position and examined on a 16-row multi-slice spiral computed tomography (MDCT) scanner (Toshiba-MEC CT3; HiSpeed, GE Medical Systems) or a 64-row MDCT scanner (Brilliance 64, Philips Medical Systems). The scanning parameters used for 16-, and 64-row MDCT scanners were detector configuration of $16 \times 0.75 \mathrm{~mm}$ and $64 \times 0.625 \mathrm{~mm}$, respectively, slice thickness of $5 \mathrm{~mm}$, table speed of 15 and $40 \mathrm{~mm} /$ rotation, respectively, rotation time of $0.5 \mathrm{~s}$, effective $\mathrm{mAs}$ of 200 , tube voltage of $120 \mathrm{kVp}$, and matrix size of $512 \times 512$. The images of the portal venous phase were used for volume measurements. Volume measurement was performed using the CT image post-processing software OsiriX v7.5.1 (Pixmeo, Switzerland).

Measurable lesions were defined using the following criteria: primary lesion covering at least four consecutive CT scanning layers and measurable lymph nodes covering at least four CT scanning layers or those greater than $15 \mathrm{~mm}$ in the short-axis diameter at the maximum cross-section.
In the portal venous phase, the enclosed area along the edge of the primary tumor or lymph node was considered a region of interest (ROI, Figure 2). The ROI area was calculated automatically using OsiriX software. The volume of the target lesion was calculated by multiplying the slice thickness of the CT scan by the sum of each ROI area using the following formula:

$$
\mathrm{V}=\sum_{n=1}^{N} \mathrm{~S}_{n} \cdot D
$$

(The layers of the target lesions covered in the CT scan were defined as $\mathrm{N}$, the ROI area for each layer was defined as Sn, slice thickness was defined as D, and target lesion volume was defined as V).

The number of measurable lesions was limited to a maximum of total five. The total volume was calculated by summing the volumes of the primary lesion and target lymph nodes. The percentage volume reduction rate (VRR) was calculated using the following equation:

$$
\begin{aligned}
\mathrm{VRR}= & {\left[\left(\begin{array}{l}
\text { pre }- \text { chemotherapy total } \\
\text { volume }
\end{array}\right)-\left(\begin{array}{l}
\text { post }- \text { chemotherapy total } \\
\text { volume }
\end{array}\right)\right] } \\
& /(\text { pre }- \text { chemotherapy total volume }) \times 100 \%
\end{aligned}
$$



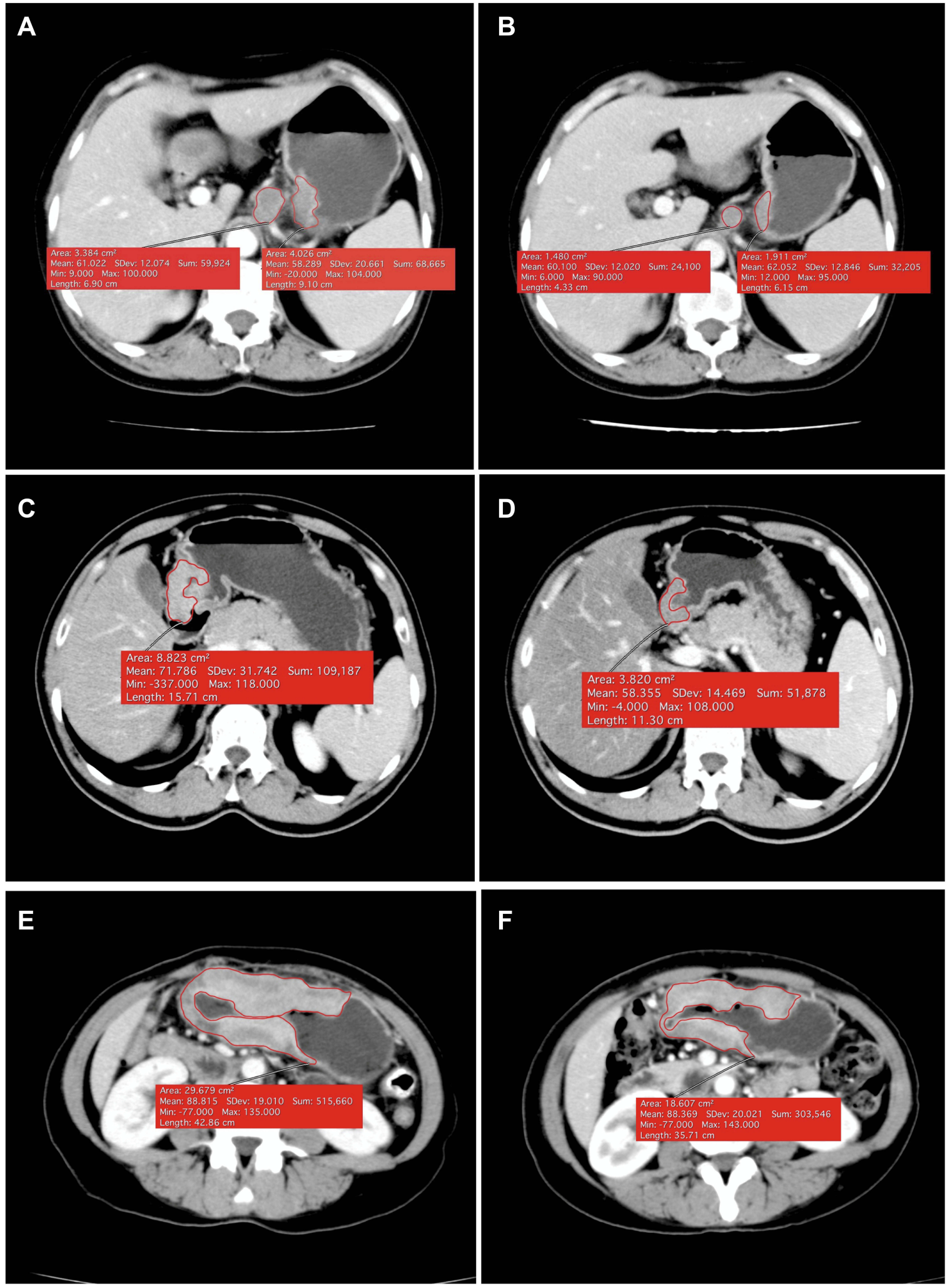

Figure 2 The CT volume measurement of the primary lesions and lymph node before and after neoadjuvant chemotherapy (NAC). Case I: the total volume was $25.3 \mathrm{~cm}^{3}$ (primary lesion $21.1 \mathrm{~cm}^{3}$, the index lymph node $4.2 \mathrm{~cm}^{3}$ ) before chemotherapy (A), and decreased to $15.0 \mathrm{~cm}^{3}$ (B) after NAC (primary lesion $13.1 \mathrm{~cm}^{3}$, the index lymph node $1.9 \mathrm{~cm}^{3}$ ), the percentage volume reduction rate (VRR) was $40.7 \%$. Case 2: the total volume was $68.6 \mathrm{~cm}^{3}$ before chemotherapy (C), and decreased to $20.8 \mathrm{~cm}^{3}$ (D) after NAC, the VRR was $69.7 \%$. Case 3: the total volume was $171.9 \mathrm{~cm}^{3}$ before chemotherapy $(\mathbf{E})$, and decreased to $101.6 \mathrm{~cm}^{3}(\mathbf{F})$ after NAC, the VRR was $40.9 \%$. 


\section{Follow-Up}

All patients returned for follow-up every 3-6 months for the first 2 years, every $6-12$ months during years $3-5$, and annually thereafter. Standard follow-up included complete blood count, chemistry profile, and tumor marker measurements and endoscopic and radiological imaging examinations (including CT, magnetic resonance imaging, and positron emission tomography-CT if necessary).

\section{Statistical Analysis}

Quantitative values, which were analyzed by the MannWhitney $U$-test, were expressed as means \pm the standard deviation. Categorical variables were expressed as absolute and relative frequencies (count and percentage) by the $\chi^{2}$ test. Thirty patients were randomized to investigate the interobserver variability of the tumor volume. Volumetric measurements for these patients were independently performed by two experienced doctors. The data were compared using the Wilcoxon test and Spearman correlation analysis.

The diagnostic accuracy of VRR in predicting the prognosis of patients receiving NAC was evaluated by receiver operating characteristic (ROC) analysis. The optimal cutoff VRR for differentiating favorable from unfavorable prognosis was defined as the point on the ROC curve closest to the $0 \%$ false-positive and $100 \%$ true-positive mark. Patients who survived more than 3 years were considered to have had a favorable prognosis, and a survival of less than 3 years was considered an unfavorable prognosis. The area under the ROC curve and the corresponding 95\% confidence interval (CI) were determined accordingly.

OS was defined as the time from the beginning of chemotherapy to death from any cause. Patients, who were alive or lost to follow-up on June 2, 2017 were censored for the analysis of OS. The Kaplan-Meier method was used for calculation of survival time, and the resulting survival curves were compared by the Log-rank test. Cox's proportional hazards model was used for multivariate analysis.

All statistical tests were two-sided, with $\mathrm{P}$ values $\leq 0.05$ considered to indicate statistical significance. Statistical analyses were performed using SPSS version 19.0 (IBM, Armonk, NY, USA).

\section{Results}

\section{Clinical and Pathological Characteristics}

A total of 117 eligible patients, consisting of 83 males and 34 females, with a median age of 60 years (range 37-80 years) were included in this study. The chemotherapy regimen was FOLFOX in 49 (41.9\%) patients, SOX in 45 (38.5\%) patients, and XELOX in $23(19.6 \%)$ patients. The median number of NAC cycles was 3 (range 2-6). The primary lesion was located in the upper, middle, and lower third of the stomach in $27(23.1 \%), 34(29.0 \%)$ and $54(46.2 \%)$ patients, respectively. Multiple regions were involved in $2(1.7 \%)$ patients. Fifty-three (45.3\%) patients received distal gastrectomy, 57 (48.7\%) received total gastrectomy, and 7 (6.0\%) received combined resection. Following surgery, $100(85.5 \%)$ patients received postoperative chemotherapy. According to the AJCC TNM staging system (7th edition), 9 (7.7\%), 22 (18.8\%), and 76 (64.9\%) patients were categorized as stage I, II, and III, respectively. Five (4.3\%) patients exhibited complete pathological tumor regression after NAC. Of the remaining $5(4.3 \%)$ patients, who could not be classified, 3 were staged as ypT0N1M0 and 2 as ypT0N2M0.

\section{Interobserver Variability}

The tumor volumes before and after chemotherapy were independently assessed by two physicians. There were no significant differences between the two physicians in terms of the tumor volume before NAC $\left(44.25 \mathrm{~cm}^{3}\right.$ vs $45.50 \mathrm{~cm}^{3}$, $p=0.43)$, tumor volume after NAC $\left(28.59 \mathrm{~cm}^{3}\right.$ vs $31.40 \mathrm{~cm}^{3}$, $p=0.14)$ or the VRR $(37.2 \%$ vs $33.6 \%, p=0.31)$. Spearman correlation coefficients for tumor volume before NAC, tumor volume after NAC, and VRR were $0.94(p<0.001), 0.88$ $(p<0.001)$, and $0.88(p<0.001)$ respectively, indicating significant reproducibility between the two observers.

\section{CT Volumetry Analysis}

Volumetric analysis revealed a mean tumor volume of $53.8 \pm$ $32.6 \mathrm{~cm}^{3}$ before NAC, which decreased to $35.9 \pm 28.4 \mathrm{~cm}^{3}$ after NAC $(p<0.001)$, with an area under the ROC curve of 0.76 (95\% CI, 0.68-0.85). According to ROC analysis, the optimal cut-off VRR was $31.95 \%$, with a sensitivity of $70.4 \%$ and a specificity of $71.7 \%$ (Figure 3 ). According to this cut-off level, patients were divided into the VRR-High (VRR $\geq 31.95 \%, \mathrm{n}=63$ ) and VRR-Low group (VRR $<31.95 \%, \mathrm{n}=54)$.

The clinical characteristics, including sex, age, chemotherapy regimen, surgical procedure, and primary tumor site, were not significantly different between the VRR-High and VRR-Low groups (Table 1). The proportion of patients with pathological $\mathrm{T}_{0-1}$ stage was $20.6 \%$ in the VRR-High group and $5.6 \%$ in the VRR-Low group. The proportion of patients with pathological $\mathrm{N}_{0}$ stage was $36.5 \%$ in the VRRHigh group and $11.1 \%$ in the VRR-Low group. In addition, 


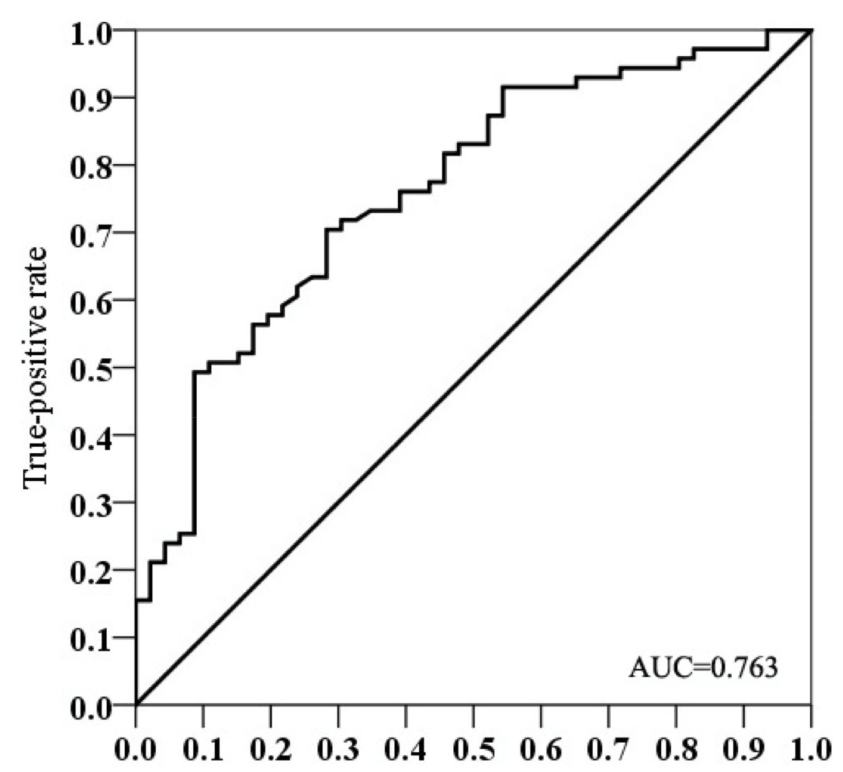

False-positive rate

Figure 3 Receiver operating characteristic (ROC) analysis of the volume reduction rate (VRR). The area under the curve was 0.763 . When the optimal cut-off level of VRR was determined to be $31.95 \%$, a sensitivity of $70.4 \%$ and a specificity of $71.7 \%$ were achieved.

four patients in the VRR-High group exhibited complete pathological tumor regression after NAC, compared with only one patient in the VRR-Low group (Table 1).

\section{Survival Analysis}

The median follow-up interval was 53.0 months (range 7-120 months). Among all patients, the 3- and 5-year survival rates were $61.5 \%(95 \% \mathrm{CI}, 52.68-70.32)$ and $48.7 \%$ (95\% CI, 39.68-57.72), respectively. The VRRLow group exhibited a worse prognosis compared with the VRR-High group (HR, 2.85; 95\% CI, 1.69-4.82, $p<0.001)$, with 3-year survival rates of $40.7 \%(95 \% \mathrm{CI}$, 27.57-53.83) and 79.4\% (95\% CI, 69.4-89.40) and 5-year survival rates of $31.5 \%(95 \% \mathrm{CI}, 19.15-43.85)$ and $63.5 \%$ (95\% CI, 51.54-75.46), respectively (Figure 4). The VRR and ypN stage were identified as independent prognostic factors in Cox's proportional hazards models (Table 2).

\section{Discussion}

NAC is widely regarded as a successful therapeutic option for AGC. Accurate and timely evaluation of the tumor response to NAC is of critical importance when making surgical decisions and may help to determine the postoperative chemotherapy regimens. ${ }^{19,20}$ Therefore, how to evaluate the tumor response to NAC is a critical issue. Until now, the common tumor response evaluation
Table I The Clinical and Pathological Characteristics of the VRR-High and VRR-Low Group

\begin{tabular}{|c|c|c|c|}
\hline & $\begin{array}{l}\text { VRR-High } \\
(\mathrm{N}=63)\end{array}$ & $\begin{array}{l}\text { VRR-Low } \\
(\mathrm{N}=54)\end{array}$ & P value \\
\hline $\begin{array}{l}\text { Gender } \\
\text { Male } \\
\text { Female }\end{array}$ & $\begin{array}{l}41 \\
22\end{array}$ & $\begin{array}{l}42 \\
12\end{array}$ & 0.13 \\
\hline $\begin{array}{l}\text { Age } \\
\quad \leq 60 \text { years } \\
>60 \text { years }\end{array}$ & $\begin{array}{l}32 \\
31\end{array}$ & $\begin{array}{l}29 \\
25\end{array}$ & 0.75 \\
\hline $\begin{array}{l}\text { Chemotherapy Regimen } \\
\text { FOLFOX } \\
\text { SOX } \\
\text { XELOX }\end{array}$ & $\begin{array}{l}28 \\
24 \\
11\end{array}$ & $\begin{array}{l}21 \\
21 \\
12\end{array}$ & 0.76 \\
\hline $\begin{array}{l}\text { Surgery } \\
\text { Total gastrectomy } \\
\text { Distal gastrectomy } \\
\text { Combined resection }\end{array}$ & $\begin{array}{l}30 \\
31 \\
2\end{array}$ & $\begin{array}{l}27 \\
22 \\
5\end{array}$ & 0.32 \\
\hline $\begin{array}{l}\text { Primary tumor site } \\
\text { Upper } \\
\text { Middle } \\
\text { Lower } \\
\text { Multiple regions involved }\end{array}$ & $\begin{array}{l}15 \\
19 \\
29 \\
0\end{array}$ & $\begin{array}{l}12 \\
15 \\
25 \\
2\end{array}$ & 0.49 \\
\hline $\begin{array}{l}\text { yрT stage } \\
\text { урT0-I } \\
\text { урТ2-3 } \\
\text { урT4 }\end{array}$ & $\begin{array}{l}13 \\
10 \\
40\end{array}$ & $\begin{array}{l}3 \\
2 \\
49\end{array}$ & 0.003 \\
\hline $\begin{array}{l}\text { ypN stage } \\
\text { ypNo } \\
\text { ypNI } \\
\text { ypN2 } \\
\text { ypN3 }\end{array}$ & $\begin{array}{l}23 \\
14 \\
17 \\
9\end{array}$ & $\begin{array}{l}6 \\
14 \\
15 \\
19\end{array}$ & 0.005 \\
\hline $\begin{array}{l}\text { yPTNM stage* } \\
\text { PCR } \\
\text { I } \\
\text { II } \\
\text { III }\end{array}$ & $\begin{array}{l}4 \\
8 \\
16 \\
31\end{array}$ & $\begin{array}{l}1 \\
1 \\
6 \\
45\end{array}$ & 0.003 \\
\hline
\end{tabular}

Notes: $\dagger \chi 2$ test; VRR-Low: volume reduction rate $<31.95 \%$; VRR-High: volume reduction rate $\geq 31.95 \%$. *Five patients could not be classified: three patients were TONIM0, and two were TON2MO.

Abbreviations: yp, yield pathological; $\mathrm{PCR}$, pathological complete regression; FOLFOX, oxaliplatin plus 5-fluorouracil plus leucovorin; SOX, oxaliplatin plus S-I; XELOX, oxaliplatin plus capecitabine.

methods in clinical include RECIST, JCGC, and histopathologic tumor regression; however, certain limitations must be considered when using these methods. The RECIST are not recommended for evaluating primary gastric cancer lesions due to the irregular tumor shape, while the JCGC criteria do not address metastatic lesions, including lymph nodes. Therefore, the validity of these two methods in evaluating gastric cancer responses to 


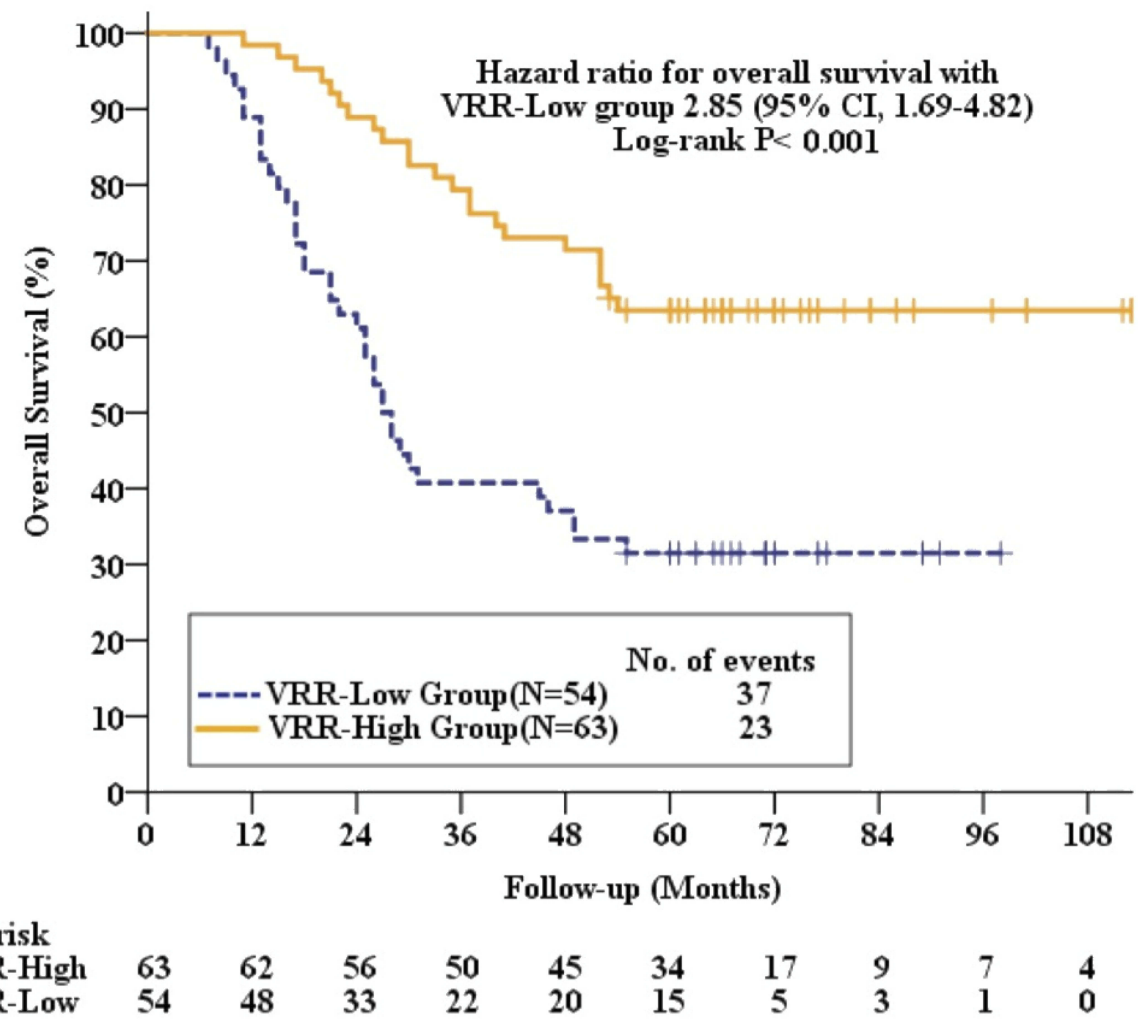

Figure 4 Survival analysis of the VRR-High group and VRR-Low group. VRR-Low group had worse survival rates compared with VRR-High group (HR, 2.85; 95\% CI, I.69-4.82, $\mathrm{P}<0.00 \mathrm{I})$.

chemotherapy remains uncertain and inconsistent. In a recent study, Kurokawa et al found that histological evaluation was a more effective assessment of the treatment response than radiological evaluation, and the OS of responders was significantly longer than that of nonresponders evaluated by histological criteria. ${ }^{21}$ Despite these findings, histopathological tumor regression was not identified as an independent prognostic factor, even though it was found to be associated with survival in a large retrospective study of esophagogastric cancer. ${ }^{22}$

In recent years, $\mathrm{CT}$ volumetry has garnered significant attention as a tool for evaluating tumor responses to neoadjuvant treatment in gastrointestinal cancers. The VRR was found to be superior to the RECIST for predicting the pathological response of rectal cancer treated with neoadjuvant chemoradiation, ${ }^{23}$ while CT volumetry predicted the long-term survival of esophageal cancer patients treated with NAC followed by surgical resection. ${ }^{24}$ However, only a few studies have used CT volumetry to assess the tumor response to NAC in gastric cancer. One study showed that patients with a VRR greater than $35.6 \%$ at 8 weeks after NAC could be categorized as pathologic responders with $100 \%$ sensitivity. ${ }^{15}$ This cut-off level was much higher than that seen in a different study, in which only patients with a VRR greater than $14.8 \%$ were considered responders. ${ }^{16}$ Nevertheless, the sample sizes of both studies were small, and the correlation of CT volumetry with long-term survival was not investigated.

In the present study, no significant interobserver variability in the measurements of CT tumor volumetry was found between the two observers, which indicated that CT volumetry was reproducible. Our sample size (117 patients) makes this the largest study investigating the use of CT volumetry to evaluate the tumor response to NAC in gastric cancer to date. The median follow-up time was 53.0 months (range 7-120 months). The cut-off VRR was determined to be $31.95 \%$ by ROC analysis and was used to identify $63(53.8 \%)$ patients as responders. Meanwhile, the maximum number of measurable lesions in this study was five, which was in accordance with the RECIST. Furthermore, the cut-off VRR of $31.95 \%$ was very close to the definitive endpoint for a partial response ( $\geq 30 \%$ decrease in the sum of the target lesion diameters) used in the RECIST.

Validated prognostic factors for patients with AGC treated with NAC followed by $\mathrm{R} 0$ resection have not been established. A meta-analysis of patient outcomes 
Table 2 Univariate and Multivariate Analyses of Prognostic Factors

\begin{tabular}{|c|c|c|c|c|c|}
\hline \multirow[t]{3}{*}{ Prognostic Factor } & \multirow[t]{3}{*}{ No. } & \multicolumn{4}{|l|}{ Survival } \\
\hline & & \multicolumn{2}{|l|}{ Univariate } & \multicolumn{2}{|l|}{ Multivariable } \\
\hline & & HR $(95 \% \mathrm{Cl})$ & $P$ value & HR (95\% Cl) & $P$ value \\
\hline \multicolumn{6}{|l|}{ Age } \\
\hline$\leq 60$ & 61 & I [Reference] & & ND & \\
\hline$>60$ & 56 & $0.73(0.44-1.21)$ & 0.22 & ND & \\
\hline \multicolumn{6}{|l|}{ Gender } \\
\hline Male & 83 & I[Reference] & & ND & \\
\hline Female & 34 & $1.61(0.95-2.73)$ & 0.07 & ND & \\
\hline \multicolumn{6}{|l|}{ Tumor site } \\
\hline Upper & 27 & I [Reference] & & ND & \\
\hline Middle & 34 & $1.80(0.86-3.80)$ & 0.12 & ND & \\
\hline Lower & 54 & $1.53(0.76-3.07)$ & 0.23 & ND & \\
\hline Multiple involved & 2 & $5.61(1.23-25.63)$ & 0.03 & ND & \\
\hline \multicolumn{6}{|l|}{ NAC regimen } \\
\hline FOLFOX & 49 & I [Reference] & & ND & \\
\hline sox & 45 & $0.62(0.34-1.12)$ & 0.11 & ND & \\
\hline XELOX & 23 & $1.13(0.59-2.17)$ & 0.72 & ND & \\
\hline \multicolumn{6}{|l|}{ Gastrectomy } \\
\hline Distal & 53 & I [Reference] & & ND & \\
\hline Total & 57 & $0.97(0.57-1.63)$ & 0.89 & ND & \\
\hline Combined resection & 7 & $1.47(0.5 \mathrm{I}-4.20)$ & 0.47 & ND & \\
\hline \multicolumn{6}{|l|}{ ур T stage } \\
\hline $\mathrm{ypT}_{0-1}$ & 16 & I [Reference] & & I [Reference] & \\
\hline yp $_{2-3}$ & 12 & $4.75(0.96-23.56)$ & 0.06 & $2.04(0.40-10.55)$ & 0.40 \\
\hline $\mathrm{ypT}_{4}$ & 89 & $6.95(1.69-28.58)$ & 0.007 & $2.13(0.50-9.13)$ & 0.31 \\
\hline \multicolumn{6}{|l|}{ ypN stage } \\
\hline $\mathrm{yp}_{\mathrm{p}}$ & 29 & I [Reference] & & I [Reference] & \\
\hline$y_{p} N_{1}$ & 28 & $8.11(1.81-36.24)$ & 0.006 & $6.10(1.33-27.95)$ & 0.02 \\
\hline yp $\mathrm{N}_{2}$ & 32 & 14.52(3.40-60.09) & $<0.001$ & $10.58(2.42-46.37)$ & 0.002 \\
\hline yp $N_{3}$ & 28 & $35.28(8.28-150.32)$ & $<0.001$ & $23.24(5.23-103.28)$ & $<0.001$ \\
\hline \multicolumn{6}{|l|}{ VRR } \\
\hline VRR-High & 63 & I [Reference] & & I [Reference] & \\
\hline VRR-Low & 54 & $2.85(1.69-4.82)$ & $<0.001$ & $1.87(1.07-3.29)$ & 0.03 \\
\hline
\end{tabular}

Notes: VRR-Low: VRR<31.95\%; VRR-High: VRR $\geq 31.95 \%$

Abbreviations: No, number; HR, hazard ratio; Cl, confidence interval; ND, no data; NAC, neoadjuvant chemotherapy; yp, yield pathological; VRR, volume reduction rate; FOLFOX, oxaliplatin plus 5-fluorouracil plus leucovorin; SOX, oxaliplatin plus S-I; XELOX, oxaliplatin plus capecitabine.

found that ypN stage, resection status, and age were all independent predictors for patients who receive NAC, but ypT stage was not. ${ }^{25}$ Although ypN and ypT stages were strongly associated with OS in the univariate analysis in this study, only ypN stage was an independent prognostic factor, consistent with previous studies. ${ }^{6,25}$ One possible explanation is that the effects of chemotherapy made it difficult to assess the influence of ypT stage on prognosis.
Although the findings of our study are encouraging, several limitations should be considered when evaluating these findings. First, although the section thickness of the CT scans was $5 \mathrm{~mm}$, the small lesions $(<5 \mathrm{~mm})$ could not be assessed well compared with thin-section CT scanning. Second, although the lesions were still detected by CT after NAC, some patients achieved complete pathological regression of the tumor. Finally, despite a significantly larger sample size compared with previous studies, a much larger multi-center 
study will be necessary to fully evaluate these findings. Hence, CT tumor volumetry combined with some imaging biomarkers, ${ }^{26,27}$ such as the apparent diffusion coefficient from diffusion-weighted magnetic resonance imaging and the textural features from contrast-enhanced multidetector computed tomography, may represent a better choice for evaluating tumor response to NAC.

\section{Conclusions}

The data presented here indicate that CT volumetry is a feasible and reliable method for assessing the tumor response to NAC in patients with AGC. Patients with a VRR exceeding $31.95 \%$ after NAC would be categorized as clinical responders.

\section{Abbreviations}

NAC, neoadjuvant chemotherapy; AGC, advanced gastric cancer; VRR, volume reduction rate; ROC, receiver operating characteristic; ROI, region of interest.

\section{Acknowledgments}

This work was supported by the Major Project of Science and Technology Department of zhejiang province (grant number 2014C03040-1). The abstract of this paper was presented at the 2018 ASCO (American Society of Clinical Oncology) Annual Meeting as an online publication with interim findings. The poster's abstract was published in "Meeting Abstract" in JOURNAL OF CLINICAL ONCOLOGY (DOI: 10.1200/JCO.2018.36.15_suppl.e16061).

\section{Disclosure}

The authors declare that they have no conflict of interest.

\section{References}

1. Torre LA, Bray F, Siegel RL, Ferlay J, Lortet-Tieulent J, Jemal A. Global cancer statistics, 2012. CA Cancer J Clin. 2015;65(2):87-108. doi:10.3322/caac. 21262

2. Sasako M, Sano T, Yamamoto S, et al. D2 lymphadenectomy alone or with para-aortic nodal dissection for gastric cancer. $N$ Engl J Med. 2008;359(5):453-462. doi:10.1056/NEJMoa0707035

3. Bang YJ, Kim YW, Yang HK, et al. Adjuvant capecitabine and oxaliplatin for gastric cancer after D2 gastrectomy (CLASSIC): a Phase 3 open-label, randomised controlled trial. Lancet. 2012;379 (9813):315-321. doi:10.1016/S0140-6736(11)61873-4

4. Ychou M, Boige V, Pignon JP, et al. Perioperative chemotherapy compared with surgery alone for resectable gastroesophageal adenocarcinoma: an FNCLCC and FFCD multicenter Phase III trial. $J$ Clin Oncol. 2011;29(13):1715-1721. doi:10.1200/JCO.2010.33.0597

5. Cunningham D, Allum WH, Stenning SP, et al. Perioperative chemotherapy versus surgery alone for resectable gastroesophageal cancer. $N$ Engl J Med. 2006;355(1):11-20. doi:10.1056/NEJMoa 055531
6. Becker K, Langer R, Reim D, et al. Significance of histopathological tumor regression after neoadjuvant chemotherapy in gastric adenocarcinomas: a summary of 480 cases. Ann Surg. 2011;253(5):934-939. doi:10.1097/SLA.0b013e318216f449

7. Al-Batran SE, Hofheinz RD, Pauligk C, et al. Histopathological regression after neoadjuvant docetaxel, oxaliplatin, fluorouracil, and leucovorin versus epirubicin, cisplatin, and fluorouracil or capecitabine in patients with resectable gastric or gastro-oesophageal junction adenocarcinoma (FLOT4-AIO): results from the Phase 2 part of a multicentre, open-label, randomised phase $2 / 3$ trial. Lancet Oncol. 2016;17(12):1697-1708. doi:10.1016/S1470-2045(16)30531-9

8. Therasse P, Arbuck SG, Eisenhauer EA, et al. New guidelines to evaluate the response to treatment in solid tumors. European Organization for Research and Treatment of Cancer, National Cancer Institute of the United States, National Cancer Institute of Canada. J Natl Cancer Inst. 2000;92(3):205-216. doi:10.1093/jnci/92.3.205

9. Eisenhauer EA, Therasse P, Bogaerts J, et al. New response evaluation criteria in solid tumours: revised RECIST guideline (version 1.1). Eur J Cancer. 2009;45(2):228-247. doi:10.1016/j.ejca.2008.10.026

10. Tokunaga M, Sugisawa N, Tanizawa Y, Bando E, Kawamura T, Terashima M. The impact of preoperative lymph node size on long-term outcome following curative gastrectomy for gastric cancer. Ann Surg Oncol. 2013;20(5):1598-1603. doi:10.1245/s10434-0122699-2

11. Hallinan JT, Venkatesh SK. Gastric carcinoma: imaging diagnosis, staging and assessment of treatment response. Cancer Imaging. 2013;13:212-227. doi:10.1102/1470-7330.2013.0023

12. Li H, Chen XL, Li JR, et al. Tumor volume of resectable gastric adenocarcinoma on multidetector computed tomography: association with N categories. Clinics (Sao Paulo). 2016;71(4):199-204. doi:10.6061/clinics

13. Wang ZC, Wang C, Ding Y, Ji Y, MS Z, Rao SX. CT volumetry can potentially predict the local stage for gastric cancer after chemotherapy. Diagn Interv Radiol. 2017;23(4):257-262. doi:10. 5152/dir.2017.16517

14. Hallinan JT, Venkatesh SK, Peter L, Makmur A, Yong WP, So JB. CT volumetry for gastric carcinoma: association with TNM stage. Eur Radiol. 2014;24(12):3105-3114. doi:10.1007/s00330-014-3316-5

15. Lee SM, Kim SH, Lee JM, et al. Usefulness of CT volumetry for primary gastric lesions in predicting pathologic response to neoadjuvant chemotherapy in advanced gastric cancer. Abdom Imaging. 2009;34(4):430-440. doi:10.1007/s00261-008-9420-8

16. Beer AJ, Wieder HA, Lordick F, et al. Adenocarcinomas of esophagogastric junction: multi-detector row $\mathrm{CT}$ to evaluate early response to neoadjuvant chemotherapy. Radiology. 2006;239(2):472-480. doi:10.1148/radiol.2391050043

17. Japanese Gastric Cancer A. Japanese gastric cancer treatment guidelines 2010 (ver. 3). Gastric Cancer. 2011;14(2):113-123. doi:10.1007/ s10120-011-0042-4

18. Edge SB, Compton CC. The American Joint Committee on Cancer: the 7th edition of the AJCC cancer staging manual and the future of TNM. Ann Surg Oncol. 2010;17(6):1471-1474. doi:10.1245/s10434010-0985-4

19. Ott K, Lordick F, Herrmann K, Krause BJ, Schuhmacher C, Siewert JR. The new credo: induction chemotherapy in locally advanced gastric cancer: consequences for surgical strategies. Gastric Cancer. 2008;11 (1):1-9. doi:10.1007/s10120-007-0448-1

20. Tang L, Li ZY, Li ZW, et al. Evaluating the response of gastric carcinomas to neoadjuvant chemotherapy using iodine concentration on spectral CT: a comparison with pathological regression. Clin Radiol. 2015;70(11):1198-1204. doi:10.1016/j.crad.2015. 06.083

21. Kurokawa Y, Shibata T, Sasako M, et al. Validity of response assessment criteria in neoadjuvant chemotherapy for gastric cancer (JCOG0507-A). Gastric Cancer. 2014;17(3):514-521. doi:10.1007/ s10120-013-0294-2 
22. Schmidt T, Sicic L, Blank S, et al. Prognostic value of histopathological regression in 850 neoadjuvantly treated oesophagogastric adenocarcinomas. $\mathrm{Br} J$ Cancer. 2014;110(7):1712-1720. doi:10. 1038/bjc. 2014.94

23. Xiao J, Tan Y, Li W, et al. Tumor volume reduction rate is superior to RECIST for predicting the pathological response of rectal cancer treated with neoadjuvant chemoradiation: results from a prospective study. Oncol Lett. 2015;9(6):2680-2686. doi:10.3892/ol.2015.3101

24. Tamandl D, Gore RM, Fueger B, et al. Change in volume parameters induced by neoadjuvant chemotherapy provide accurate prediction of overall survival after resection in patients with oesophageal cancer. Eur Radiol. 2016;26(2):311-321. doi:10.1007/s00330-015-3860-7
25. Ronellenfitsch U, Schwarzbach M, Hofheinz R, et al. Predictors of overall and recurrence-free survival after neoadjuvant chemotherapy for gastroesophageal adenocarcinoma: pooled analysis of individual patient data (IPD) from randomized controlled trials (RCTs). Eur J Surg Oncol. 2017;43(8):1550-1558. doi:10.1016/j.ejso.2017.05.005

26. Giganti F, Tang L, Baba H. Gastric cancer and imaging biomarkers: part 1-a critical review of DW-MRI and CE-MDCT findings. Eur Radiol. 2019;29(4):1743-1753. doi:10.1007/s00330-018-5732-4

27. Jiang Y, Chen C, Xie J, et al. Radiomics signature of computed tomography imaging for prediction of survival and chemotherapeutic benefits in gastric cancer. EBioMedicine. 2018;36:171-182. doi:10.1016/j.ebiom.2018.09.007

\section{Publish your work in this journal}

Cancer Management and Research is an international, peer-reviewed open access journal focusing on cancer research and the optimal use of preventative and integrated treatment interventions to achieve improved outcomes, enhanced survival and quality of life for the cancer patient.
The manuscript management system is completely online and includes a very quick and fair peer-review system, which is all easy to use. Visit http://www.dovepress.com/testimonials.php to read real quotes from published authors. 